\title{
Implicações das concentrações séricas de selênio nas doenças inflamatórias intestinais: uma revisão bibliográfica
}

\author{
Implications of serum selenium concentrations on inflamatory bowel diseases: a \\ bibliographic review
}
Implicaciones de lãs concentraciones sericas de selênio em las enfermidades inflamatórias del intestino: una revisión bibliográfica

Ayla Patrícia Soares do Nascimento ${ }^{1 *}$, Emilene Maciel e Maciel ${ }^{1}$, Ana Carolina de Jesus Nunes de Oliveira$^{1}$, Cyntia Regina Lúcio de Sousa Ibiapina², Maria da Cruz Moura e Silva².

\section{RESUMO}

Objetivo: Revisar a literatura atual acerca das implicações das concentrações séricas anormais de Selênio (Se) nas Doenças Inflamatórias Intestinais. Métodos: Trata-se de uma revisão de literatura, realizada por meio de artigos publicados nos últimos dez anos (2007-2017), nas línguas portuguesa e inglesa, indexados nas bases de dados PubMed/MEDLINE, SCIELO e LILACS. Foram incluídos estudos de caso-controle, intervencionais e experimentais. Resultados: Foram selecionados 63 artigos, dos quais 9 foram analisados de forma completa. Após análises, constatou-se que 100\% dos artigos apontaram baixas concentrações de $\mathrm{Se}$ em pacientes com DII, principalmente na Doença de Crohn. Observou-se ainda, que as principais implicações disso para esta população foram: aumento da inflamação intestinal, diminuição da ação da glutationa peroxidase, diminuição da proteção contra o câncer. Considerações finais: Conclui-se que indivíduos portadores de DII apresentam baixas concentrações de Se e que este mineral exerce efeito protetor, sugerindo que intervenção terapêutica com a suplementação dietética de Se deve ser encorajada nesses pacientes.

Palavras-chave: Doenças Inflamatórias Intestinais, Selênio, Doença de Crohn, Colite ulcerativa.

\begin{abstract}
Objective: To review the current literature on the implications of abnormal serum selenium (Se) concentrations on inflammatory bowel disease. Methods: This is a literature review, conducted through articles published in the lastest ten years (2007-2017), in Portuguese and English, indexed in PubMed / MEDLINE, SCIELO and LILACS databases. Results: A total of 63 articles were selected, where 9 were analyzed entirely. After analysis, it was found that $100 \%$ of these indicated low Se concentrations in patients with IBD, especially in Crohn's disease. It was also observed that the main implications of this population were: decreased glutathione peroxidase action, decreased protection against cancer. Final considerations: It is concluded that individuals with IBD have low Se concentrations and that this mineral exerts a protective effect, suggesting that therapeutic intervention with Se dietary supplementation should be encouraged in these patients.
\end{abstract}

Keywords: Inflammatory Bowel Diseases, Selenium, Crohn disease, Colitis ulcerative.

\section{RESUMEN}

Objetivo: revisar la literatura actual sobre las concentraciones anormales de selenio en suero (Se) en la enfermedad inflamatoria intestinal. Métodos: Esta es una revisión de la literatura, realizada a través de artículos publicados en los últimos diez años (2007-2017), en portugués e inglés, indexados en las bases de datos PubMed / MEDLINE, SCIELO y LILACS. Resultados: Seleccionamos 63 artículos, 9 de los cuales

\footnotetext{
${ }_{1}$ Programa de Residência Multiprofissional. Universidade Federal do Piauí. Hospital Universitário, Teresina, Piauí. *E-mail: aylasoares10@hotmail.com

${ }^{2}$ Hospital Universitário, Universidade Federal do Piauí, Teresina, Piauí.
}

SUBMETIDO EM: 10/2019

ACEITO EM: 11/2019

PUBLICADO EM: 2/2020 
fueron completamente analizados. Después del análisis, se encontró que el 100\% de estos indicaban bajas concentraciones de Se en pacientes con Ell, especialmente en la enfermedad de Crohn. También se observó que las principales implicaciones de esta población eran: aumento de la inflamación intestinal, disminución de la acción de la glutatión peroxidasa, disminución de la protección contra el cáncer. Consideraciones finales: se concluye que las personas con Ell tienen bajas concentraciones de Se y que este mineral ejerce un efecto protector, lo que sugiere que se debe alentar la intervención terapéutica con suplementos dietéticos de Se en estos pacientes.

Palabras clave: Enfermedades inflamatorias del intestino, Selenio, Enfermedad de Crohn, Colitis ulcerosa.

\section{INTRODUÇÃO}

As Doenças Inflamatórias Intestinais (DII) são doenças caracterizadas por inflamação aguda, recorrente ou crônica que pode afetar vários segmentos do Trato Gastrointestinal (TGI), especialmente os intestinos. As DII incluem a Colite Ulcerativa (CU) e Doença de Crohn (DC). Enquanto a CU é exclusivamente restrita ao intestino grosso, a DC podeafetarqualquer segmento do TGI, desde a boca até o ânus. Os principais sintomas em geral incluem: diarreia excessiva e esteatorreia, que pode ocorrer até 20 vezes por dia,e sintomas característicos como diarreia sanguinolenta, cólicas abdominais, urgência para evacuar na CU; eliminação de muco e diarreia, dor abdominal, anorexia, emagrecimento e febre na DC (GROPPER SS e SMITH JL, 2013; SALARI P, 2011; LUCENDO AJ e REZENDE LC, 2009).

A etiologia dessas doenças é desconhecida, portanto, a prevenção ou a cura ainda não são possíveis. No entanto, de acordo com estudos epidemiológicos as causas são multifatoriais e estão relacionadas com a idade, raça e distribuição geográfica. Sabe-se que até $15 \%$ das pessoas com DII possui um parente de primeiro grau queé portador de DII. A DC é mais comum nas mulheres, já a CU é ligeiramente mais frequente nos homens. A idade máxima para o início da DC é usualmente compreendida entre 20-30 anos e para a CU entre 30-40, porém, isso não é regra e ambas podem ocorrer em qualquer fase da vida (CDC, 2017; ANANTHAKRISHNAN AN, 2015; MOLODECKY N, et al., 2012).

O aumento da incidência e da prevalência das DIl é crescente no mundo inteiro, o que possivelmente é influenciado por fatores como industrialização, estresse, tabagismo, mudanças na dieta e no estilo de vida da população. Estima-se que mais de 1 milhão de americanos possuem alguma DII, mais de 2 milhões de pessoas na Europa, e várias centenas de milhares a mais no mundo. No Brasil, a prevalência ainda é baixa, porém houve um aumento significativo da incidência nas últimas décadas, no entanto poucos são os estudos sobre os aspectos epidemiológicos das doenças inflamatórias intestinais no país (BURISCH J e MUNKHOLM P, 2015; MOLODECKY N, et al., 2012).

O estado nutricional está relacionado com a gravidade da doença, uma vez que estado nutricional inadequado compromete o sistema imune. Portanto, hábitos alimentares adequados contribuem para melhor prognóstico dos portadores da doença. O cuidado nutricional é relevante nas DII, pois atuam na prevenção e tratamento da desnutrição, assim como nas deficiências de nutrientes e alterações nos níveis de oligoelementos, como zinco, selênio (Se) e cobre que são comuns nesses pacientes (SALVIANO FN, et al., 2007). O Se é um dos antioxidantes essenciais estudado em portadores de DII, visto que sua deficiência é muito comum nesses pacientes, o que compromete o estado de defesa antioxidante do organismo (GEERLING B, et al., 2000). Nesse contexto, este estudo tem como objetivo revisar a literatura acerca das possíveis implicações das concentrações séricas anormais de Se nas DII.

\section{MÉTODOS}

Trata-se de uma de revisão de literatura, realizada por meio de pesquisas de artigos indexados nas bases de dados Medical Literature Analysis and Retrieval System Online (MEDLINE/ PubMed), Scielo Eletronic Libray Online (SCIELO) e Literatura Latino-americana e do Caribe em Ciências da Saúde (LILACS). As palavras-chave utilizadas na pesquisa bibliográfica foram: Doenças Inflamatórias Intestinais, Doença de Chron, Retocolite, Selênio, em português e suas respectivas na língua inglesa. 
Como critérios de inclusão dos estudos, foram determinados os seguintes: período de publicação de 2007 a 2017, idioma de publicação (português ou inglês) e desenho do estudo: caso-contole, intervencional ou experimental. Foram excluídos os artigos de revisão e aqueles disponíveis apenas no formato de resumo, além dos repetidos. Foram encontrados 63 artigos, dos quais apenas 9 foram incluídos nesta revisão para leitura do texto completo, os demais não se enquadraram nos critérios de busca acima descritos, portanto foram excluídos da pesquisa (Figura 1).

Figura 1 - Fluxograma da revisão de literatura.

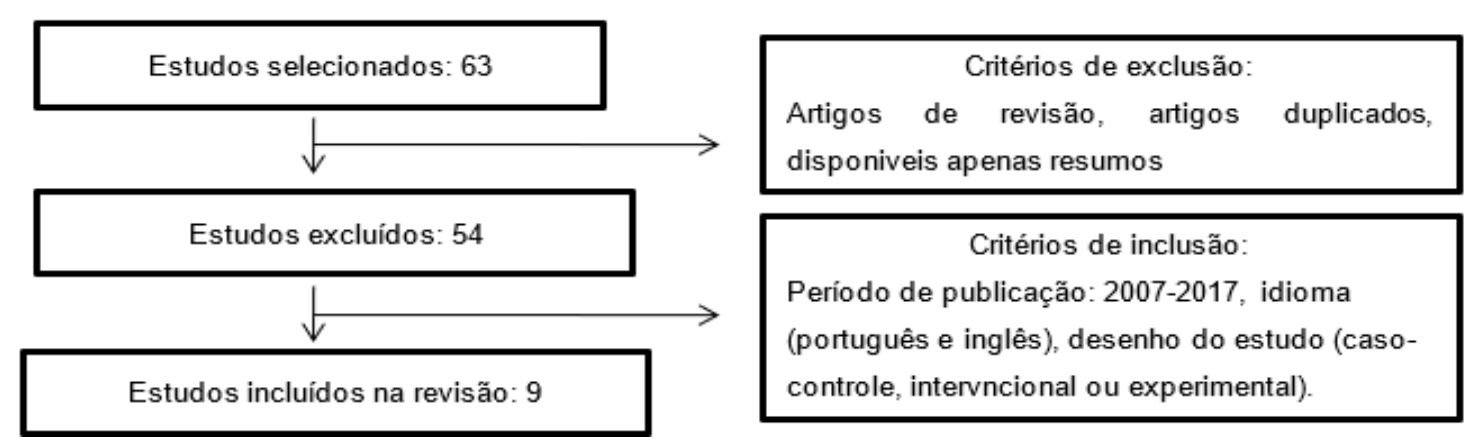

Fonte: Nascimento APS, et al., 2019.

\section{RESULTADOS E DISCUSSÃO}

Nesta revisão bibliográfica, dos 63 artigos pesquisados, 100\% $(n=9)$ deles evidenciaram baixas concentrações de Se em pacientes com DII, principalmente na Doença de Crohn. Observou-se ainda, que as principais implicações disso para esta população foram: aumento da inflamação intestinal, diminuição da ação da glutationa peroxidase e diminuição da proteção contra o câncer (Tabela 1).

\section{Selênio (Se)}

O Se é um elemento traço não metálico que foi identificado como um resíduo da oxidação do dióxido de enxofre a partir de piritas de cobre na produção de ácido sulfúrico em 1818 por Berzelius e foi denominado selênio a partir da lua (selene = lua em grego). Em 1957 Schwarz e Foltz descobriram as propriedades nutricionais essenciais do selênio, dando início a uma era de pesquisas que continuam até hoje. Assim, ao invés de uma preocupação primária com a toxicidade do Se, a atenção foi voltada para a função metabólica desse elemento e as consequências provenientes de sua deficiência (NATIONAL ACADEMY PRESS, 1983).

Esse oligoelemento é encontrado amplamente distribuído em toda a crosta terrestre e a quantidade é bem variada, podemos observar solos que apresentam conteúdos de Se com quantidades traço e até níveis tóxicos, a exemplo dos solos riquíssimos da Irlanda que chegam a atingir concentrações acima de 1.000 $\mathrm{mg} / \mathrm{kg}$. O conteúdo e biodisponibilidade nos alimentos é determinado pela concentração no solo e pela eficiência do sistema de transferência solo-planta, portanto o estado nutricional de Sedas populações humanas geralmente é geograficamente correlacionado e a biofortificação agronômica foi proposta para várias culturas e regiões, como na China para aliviar a deficiência de Se em seres humanos (WINKEL LHE, et al., 2015; NOTHSTEIN AK, et al., 2016).

O Se pode apresentar-se em duas formas e suas subdivisões. Na forma inorgânica: metálica ( $\left.\mathrm{Se}^{\circ}\right)$ ou oxiânions como selenito ( $\mathrm{SeO} 3$ 2-) e selenato ( $\mathrm{SeO} 4$ 2-), e também na forma orgânica: selenocisteína (Sec) e selenometionina (SeMet). As principais fontes de Se são carnes e peixes, ovos, produtos lácteos, cereais oleaginosas e lêvedo de cerveja. Frutas e verduras, em geral, não são boas fontes de Se, porém, alguns vegetais (alho, mostarda indiana, brócolis, couve-de-bruxelas, couve, couve-flor, repolho, cebola e alguns cogumelos) classificados como "acumuladores" de Se. A recomendação de ingestão diária para adultos de

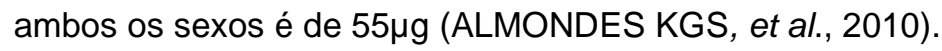

Sua absorção ocorre, em grande parte, na extremidade inferior do intestino delgado, sendo todas as suas formas facilmente absorvidas. A maior parte do selenito é absorvida no duodeno por difusão passiva, 
enquanto o selenato é ativamente absorvido no íleo por cotransporte com íons de sódio. O Se oriundo da SeMet também é absorvido no intestino delgado, com maior taxa de absorção no duodeno pelo sistema sódio dependente. A principal forma de excreção do Se é via urina, mas pode ocorrer também pelas fezes, (COMINETTI C e COZZOLINO SMF, 2009).

Em relação às funções desempenhadas pelo Se no organismo podemos destacar a sua participação na modulação dos hormônios da tireóide convertendoo T4 (tiroxina) em T3 (triiodotironina), redução do risco de doenças crônicas não transmissíveis e aumento da resistência do sistema imunológico, detoxificação de metais pesados e xenobióticos, estabilização do metabolismo do ácido araquidônico, aumenta a eficiência da vitamina $E$, e sua capacidade antioxidante, atuando como um elemento antioxidante na neutralização de radicais livres desempenhando efeito antimutagênico na prevenção do câncer relacionado com neoplasias malignas no cólon que podem estar intimamente relacionados com as DIII (COMINETTI C e COZZOLINO SMF, 2009; SILVA JF, 2015).

Abrangendo a sua função antioxidante, o Se integra o sítio ativo da enzima antioxidante glutationa peroxidase (GPx) que catalisa a oxidação da glutationa reduzida para a oxidada. A glutationa protege os lipídios das membranas e ainda de outros constituintes celulares contra a lesão oxidativa através das espécies reativas de oxigênio (ROS) (VIARO RS, et al., 2001).

$\mathrm{O}$ Se atua como um catalisador antioxidante, modulalando a expressão da via do fator nuclear $\mathrm{k} \beta$ (NF-k $\beta$ ) que influencia no desenvolvimento de inflamação e carcinogênese. A atuação do Se na inibição da carcinogênese é devido à proteção das membranas celulares contra os danos oxidativos, o que reduz a mutagenicidade de substâncias químicas cancerígenas ou afeta o metabolismo carcinogênico, portanto é importante a avaliação dos níveis de Se em pacientes portadores de DII, pois níveis reduzidos desse mineral podem ocasionar consequências, como o desenvolvimento de câncer intestinal (STOFFANELLER R e MORSE NL, 2015; BARNETT M, et al., 2010).

\section{Doenças Inflamatórias Intestinais (DII)}

As duas formas clássicas de DIl são DC e CU, ambas apresentam como sintomas diarreia, febre, anemia, intolerâncias alimentares, desnutrição, déficit de crescimento e manifestações extraintestinais como colangite esclerosante na CUe cálculos biliares na DC. A CU é delimitada à mucosa do colón e é caracterizada por melena (diarreia sanguinolenta, ulceração difusa e profunda). Ao passo que a DC possui caráter granulomatoso e pode afetar qualquer parte do TGI, com predominância no íleo terminal e colón. Os sintomas incluem diarreia, dor abdominal e massa no abdome. Essas doenças ocorrem com mais frequencia em pessoas entre 15 e 30 anos e de ambos os sexos (MAHAN LK, et al., 2013).

A prevalência é de aproximadamente 100 casos para 100.000 indivíduos na CU e 130 por 100.000 para a DC. De acordo com dados obtidos do Center for Disease Control and Prevention - CDC (2017). As DII são mais comuns em países desenvolvidos como o norte Europeu, Estados Unidos e Canadá, com crescimento recentemente no Japão, Argentina, Chile, Uruguai e nas regiões Sul e Sudeste do Brasil que abrange os estados mais socioeconomicamente desenvolvidos. Com maior frequência nas áreas urbanas e em climas do Norte. Estes dados mostram que a distribuição geográfica está ligada a fatores ambientais, tais como dieta, hábito de fumar, poluição, diferenças na exposição solar e produtos químicos (PROTÁSIO BKPF, et al., 2018).

A fisiopatologia envolve principalmente a predisposição genética e a disfunção do sistema imunológico digestivo que desencadeia respostas inflamatórias não controladas, graves e prolongadas em indivíduos geneticamente predispostos somados com uma influência ambiental desconhecida que interage com a microbiota intestinal e afeta o TGl. Este desequilíbrio imunológico provoca aumento na produção e liberaçãode citocinas inflamatórias como fator de necrose tumoral a (TNF- $\alpha$ ), interferon- $\alpha$ (IFN- $\alpha$ ), e interleucinas (IL), IL-12 e IL-17, na DC e IL-13, entre outras na retocolite ulcerativa. Outros fatores incluem infecção microbiana, estresse oxidativo e dieta inadequada (GROPPER SS e SMITH JL, 2012; SALARI P, 2011; MALIK T e MANNON P, 2012).

A predisposição genética pode ser explicada através da imunidade inata que é responsável em grande parte pelo equilíbrio imunológico no nosso organismo e, que é composta por macrófagos e células dendríticas,

REAS/EJCH | Vol.12(2) | e2101 | DOI: https://doi.org/10.25248/reas.e2101.2020 Página 4 de 9 
as quais têm a capacidade de detectar microrganismos existentes na mucosa intestinal. A superfície dessas células possui um grande número de receptores/sensores que interagem com uma série de bactérias gastrointestinais, e são classificados em duas grandes famílias: TLR (toll-like receptor) e NOD (Nucleotidebinding oligomerization domain). Quando ativados por moléculas bacterianas, estes receptores desencadeiam uma serie de reações em cadeia, que irão ocasionar uma resposta para manter o equilíbrio no epitélio da mucosa intestinal (MAHAN LK, et al, 2013; PINHO M, 2008).

Dentre as consequências que estas doenças podem ocasionar para seus portadores incluem-se, a má absorção de nutrientes, obstruções gastrointestinais parciais, alteraçõesno trânsito gastrointestinal, aversões alimentares, ressecções que podem ocasionar síndrome do intestino curto, entre outras (MAHAN LK, et al., 2013).

\section{Níveis Séricos de Selênio e DII}

As lesões no TGI decorrentes do processo inflamatório das DIl podem levar à desnutrição, devido à atividade da doença; má absorção de nutrientes, anorexia, necessidades nutricionais elevadas, estresse oxidativo, uso de medicamentos e diminuição na ingestão dos alimentos (LUCENDO AJ e REZENDE LC, 2009; GROPPER SS e SMITH JL, 2013).

As deficiências nutricionais, especialmente de vitaminas e micronutrientes essenciais, são comuns e podem ocorrer ainda no início. São ocasionadas pela inflamação crônica presente no intestino, isso porque ocorre a elevação dos requerimentos de oligoelementos antioxidantes, que são absorvidos no intestino delgado. Os mecanismos que contribuem para tais deficiências são: diminuição da ingestão de nutrientes, aumento da perda intestinal de nutrientes, má absorção, estado hipermetabólico e interações droga-nutriente (YOON SM, 2016; LUCENDO AJ e REZENDE LC, 2009).

As principais carências nutricionais que estes pacientes podem apresentar são de ferro, folato, vitamina B12; cálcio, vitamina D, vitamina $\mathrm{K}$; zinco, vitamina A e vitamina $\mathrm{C}$. Estas desordens estão associadas ao desenvolvimento de anemia, doença óssea, dificuldades de cicatrização, e risco de câncer colorretal. Pacientes com DII apresentam frequentemente deficiência de Se e de GPx, enzima antioxidante dependente de Se que reduz o estresse oxidativo e participa na manutenção da integridade epitelial intestinal; além disso, identificaram níveis séricos baixos da selenoproteína $\mathrm{P}$, que é uma proteína plasmática que transporta a maior parte do Se plasmático e é um importante antioxidante sérico (YOON SM, 2016; BARRETT CW, et al., 2013; KUDVA AK, et al., 2015).

As concentrações séricas de Se nos portadores de DII podem ser influenciadas pelo índice de massacorporal (IMC), duração e extensão e gravidade da doença, tratamento medicamentoso e cirúrgico. $O$ efeito benéfico que o Se pode exercer nas DIl's, é devido à ação combinada com a microbiota intestinal na modulação da inflamação. Em razão do aumento do Se, há uma elevação das selenoproteínas que por sua vez desativam a prostaglandina inflamatória E2 (PGE2) secretada por macrófagos, ocasionando a supressão de marcadores M1, que são pro-inflamatórios e uma regulação positiva de marcadores M2, anti-inflamatórios, diminuindo a inflamação intestinal (KUDVA AK, et al., 2015; KAUSHAL N, et al., 2014; AGUILAR-TABLADA TC, et al., 2016).

Alguns estudos mostram que a suplementação de Se em portadores de DII resulta na elevação das concentrações séricas, melhorando o perfil de deficiência e o estado antioxidante a partir da ação da GPx. Em outros, porém não foi identificado benefício com a suplementação de Se, possivelmente porque as vantagens da suplementação de Se dependem de vários fatores, como o estado normal de selênio, o cunho genético (existência ou não de polimorfismos nos genes de selenoproteínas), presençade câncer, assim como a conversão metabólica e a dose de Se suplementada (GEERLING B, et al., 2000; AGUILAR-TABLADA TC, et al., 2016; STEINBRENNER H, et al., 2013). 
Tabela 1 - Implicações de baixas concentrações de Se em indivíduos com doença inflamatória intestinal.

\begin{tabular}{|c|c|c|c|}
\hline Autor (ano) & Desenho do estudo & Objetivos & Conclusões \\
\hline $\begin{array}{c}\text { Han YM, et al. } \\
\text { (2017) }\end{array}$ & Retrospectivo & $\begin{array}{l}\text { Avaliar a prevalência de deficiência de } \\
\text { micronutrientes (vitamina D, zinco e Se) em } \\
\text { pacientes coreanos com DIl e os fatores } \\
\text { desencadeantes destas deficiências. }\end{array}$ & $\begin{array}{l}\text { Houve quantidade considerável de pacientes com } \\
\text { deficiências de vitamina } D \text {, zinco e Se. Os fatores } \\
\text { desencadeantes da carência de Se foram: sexo feminino e } \\
\text { níveis baixos de albumina sérica, uso de corticosteróides e } \\
\text { níveis séricos de PCR elevados. }\end{array}$ \\
\hline $\begin{array}{l}\text { Aguilar-Tablada } \\
\text { TC, et al. } \\
(2016)\end{array}$ & Caso- controle & $\begin{array}{l}\text { Determinar as concentrações de Se sérico em } \\
\text { pacientes com CU e DC e comparar com } \\
\text { indivíduos saudáveis. }\end{array}$ & $\begin{array}{l}\text { Concentrações de Se foram menores nos portadores de DII } \\
\text { do que nos controles e menores na } D C \text { que na CU. Os níveis } \\
\text { de Se, foram menores nos pacientes em uso de } 5 \text {-acetil } \\
\text { salicílico + corticóides + imunossupressores. O Se sérico foi } \\
\text { significativamente menor em pacientes desnutridos (IMC } \leq \\
18,5 \text { ) do que nos obesos (IMC } \geq 30 \text { ). }\end{array}$ \\
\hline $\begin{array}{c}\text { Manal MY, et al. } \\
(2015)\end{array}$ & $\begin{array}{l}\text { Prospectivo } \\
\text { intervencional }\end{array}$ & $\begin{array}{l}\text { Avaliar o efeito da suplementação de Se na } \\
\text { proteína C-reativa (PCR), calprotectina } \\
\text { (marcador na detecção da DII) e atividade da } \\
\text { doença. }\end{array}$ & $\begin{array}{l}\text { A suplementação de Se reduziu significativamente a PCR e } \\
\text { a calprotectina sérica e aumentou o nível sérico de Se, } \\
\text { porém a atividade da doença não sofreu alteração. }\end{array}$ \\
\hline $\begin{array}{l}\text { Kaushal N, et al. } \\
\qquad(2014)\end{array}$ & $\begin{array}{l}\text { Experimental } \\
\text { (indução) } \\
\text { In vivo }\end{array}$ & $\begin{array}{l}\text { Examinar o papel das selenoproteínas de } \\
\text { macrófagos na progressão e severidade da } \\
\text { doença, através de avaliação histopatológica, } \\
\text { expressão de genes pró e anti-inflamatórios e } \\
\text { modulação de metabólitosde PGE2 na urina e } \\
\text { no plasma. }\end{array}$ & $\begin{array}{l}\text { As selenoproteínas têm papel anti-inflamatório na colite } \\
\text { experimental, onde a inativação metabólica das PGE2 } \\
\text { parece ser o principal regulador da patogênese da doença; } \\
\text { níveis dietéticos mais elevados de Se foram responsáveis } \\
\text { pela inativação destas PGE2. }\end{array}$ \\
\hline $\begin{array}{l}\text { Pinto MAS, et al. } \\
\qquad(2013)\end{array}$ & Caso- controle & $\begin{array}{l}\text { Avaliar a atividade enzimática da GPx, a } \\
\text { concentração desta na mucosa inflamada e não } \\
\text { inflamada na DC e as concentrações } \\
\text { sanguíneas e plasmáticas de Se na DC e } \\
\text { comparar com controles saudáveis. }\end{array}$ & $\begin{array}{l}\text { Não houve diferenças significativas entre os grupos, quanto } \\
\text { às concentrações de Se plasmático e sangue total. Porém, } \\
\text { a mucosa inflamada na DC apresentou menor atividade de } \\
\text { GPx em relação aos outros grupos; a diminuição da } \\
\text { atividade da GPx intestinal na mucosa inflamada não foi } \\
\text { associada à ingestão de Se na dieta e concentrações } \\
\text { plasmáticas dele. }\end{array}$ \\
\hline
\end{tabular}




\begin{tabular}{|c|c|c|c|}
\hline $\begin{array}{l}\text { Barrett CW, et al. } \\
\qquad(2013)\end{array}$ & $\begin{array}{l}\text { Experimental } \\
\text { (indução) } \\
\text { In vivo }\end{array}$ & $\begin{array}{l}\text { Determinar a influência da deficiência de Se } \\
\text { dietético na gravidade da lesão colônica } \\
\text { experimental induzida por Sulfato de Dextrano } \\
\text { de Sódio (DSS), em camudongos. }\end{array}$ & $\begin{array}{l}\text { O Se provocou remissão de lesão colônica induzida por } \\
\text { DSS, protege contra a progressão de tumores, sua carência } \\
\text { induz aumento de lesão e inflamação do cólon. }\end{array}$ \\
\hline $\begin{array}{l}\text { Krehl S, et al. } \\
\qquad(2012)\end{array}$ & $\begin{array}{l}\text { Experimental } \\
\text { (indução) } \\
\text { In vivo }\end{array}$ & $\begin{array}{l}\text { Analisar a inflamação intestinal induzida por } \\
\text { tratamento com azoxometano (composto } \\
\text { carcinógeno e neurotóxico) e DSS em } \\
\text { camundongos suplementados r com } \\
\text { selenometionina como fonte de Se }\end{array}$ & $\begin{array}{l}\text { O Se protegeu contra inflamação intestinal, desenvolvimento } \\
\text { e progressão do câncer, revertendo os efeitos negativos de } \\
\text { sua deficiência na presença de inflamação. }\end{array}$ \\
\hline $\begin{array}{l}\text { Gentschew L, et } \\
\quad \text { al. (2012) }\end{array}$ & Caso- controle & $\begin{array}{l}\text { Avaliar a relação dos níveis séricos de Se na } \\
\text { indução do risco da DC }\end{array}$ & $\begin{array}{l}\text { Indivíduos com níveis séricos de Se baixo possuíam risco } \\
\text { significativamente maior de desenvolverem DC comparados } \\
\text { aos de níveis elevado e médio. }\end{array}$ \\
\hline $\begin{array}{l}\text { Barger JL, et al. } \\
\qquad(2011)\end{array}$ & $\begin{array}{c}\text { Abordagem } \\
\text { nutrigenômica }\end{array}$ & $\begin{array}{l}\text { Avaliar os efeitos de três formas diferentes de } \\
\text { Se (L-selenometinina (SM), selenite de sódio } \\
\text { (SS) ou Se de fermento (YS) em quatro tecidos } \\
\text { de camundongos suplementados com dieta } \\
\text { pobre e adequada emSe. }\end{array}$ & $\begin{array}{l}\text { Todas as formas de Se utilizadas foram capazes de corrigir } \\
\text { alterações na expressão de genes que codificam proteínas } \\
\text { de ligação do Se e também corrigiram a atividade da GPx, } \\
\text { isto é, diferentes formas de Se possuem capacidade para } \\
\text { corrigir uma deficiência deste mineral. }\end{array}$ \\
\hline
\end{tabular}

Fonte: Nascimento APS, et al., 2019. 


\section{CONSIDERAÇÕES FINAIS}

Os estudos avaliados demonstraram baixas concentrações de Se em pacientes com DII, principalmente na DC, Observou-se ainda, que as principais implicações disso para esta população foram: aumento da inflamação intestinal, diminuição da ação da glutationa peroxidase e diminuição da proteção contra o câncer. Contudo, mais estudos são necessários para compreensão da relação causa-efeito do status baixo de Se em pacientes com DII, de como o Se protege contra a tumorigênese, para quais tipos de câncer é mais importante e quais os níveis dietéticos mais adequados. Os achados dos estudos também sugerem que intervenção terapêutica com suplementação dietética de Se deve ser encorajada em pacientes com deficiência comprovada.

\section{REFERÊNCIAS}

1. AGUILAR-TABLADA TC, et al. Ulcerative Colitis and Crohn's Disease are associated with decreased serum selenium concentrations and increased cardiovascular risk. Nutrients, 2016; 8(12):780.

2. ALMONDES KGS, et al. The role of seleno proteins in câncer. Rev da Associação Médica Brasileira, 2010;56(40): 484-488.

3. ANANTHAKRISHNAN AN. Epidemiology and risk factors for IBD. Nat Rev Gastroenterol Hepatol, 2015.

4. BARGER JL. et al. Gene expression profiling reveals differential effects of sodium selenite, seleno methionine, and yeast-derived selenium in the mouse. Genes \&amp; Nutrition, 2011; 7(2):155-165.

5. BARNETT M, et al. Investigating micronutrients and epigenetic mechanisms in relation to inflammatory bowel disease. Mutat Res., 2010; 690:71-80.

6. BARRETT CW. et al. Dietary selenium deficiency exacerbates DSS-induced epithelial injury and AOM/DSS-induced tumorigenesis. PLoS One, 2013; 8: e67845.

7. BURISCH J, MUNKHOLM P. The epidemiology of inflammatory bowel disease. Scandinavian Journal of Gastroenterology, 2015; 50(8): 942-951.

8. CDC - Center for Disease Control and Prevention. Inflammatory Bowel Disease (IBD). Epidemiology of IBD.

9. COMINETTI C, COZZOLINO SMF. Funções plenamente reconhecidas denutrientes: Selênio. Série de publicações ILSI. Brasil, 2009; 8:1-20.

10. GEERLING B, et al. Comprehensive nutritional status in recently diagnosed patients with inflammatory bowel disease compared with population controls. Eur J Clin Nutr. 2000; 54(6):514-21.

11. GENTSCHEW L, et al. Selenium, seleno protein genes and Crohn's disease in a case -control population from Auckland. Nutrients, 2012; 4:1247-1249.

12. GROPPER SS, SMITH JL. Advanced Nutrition and Human Metabolism, Sixth Edition. Wadsworth, Cengage Learning, 2013.

13. HAN YM, et al. Risk Factors for Vitamin D, Zinc, and Selenium Deficiencies in Korean Patients with Inflammatory Bowel Disease. Gut and Liver, 2017; 11(3):363-369.

14. KAUSHAL N, et al. Crucial role of macrophage seleno proteins in experimental colitis. J. Immunol., 2014; 193:36833692.

15. MAHAN LK, et al. Krause: Alimentos, Nutrição e Dietoterapia.13 ed. Rio de Janeiro: Elsevier, 2013.

16. KREHL S, et al. Glutathione peroxidase-2 and selenium decreased inflammation and tumors in a mouse model of inflammation associated carcinogenesis whereas sulforaphane effects differed with selenium supply. Carcinogenesis, 2012; 33:620-628.

17. KUDVA AK, et al. Selenium and inflammatory disease. Am. J. Physiol. Gastroenterol. Liver Physiol., 2015; 309: G71G77.

18. LUCENDO AJ, REZENDE LC. Importance of nutrition in inflammatory bowel disease. World J Gastroenterol., 2009.

19. MALIK T, MANNON P. Inflammatory bowel diseases: emerging therapies and promising molecular targets. Front Biosci, 2012; 1(4):1172-89.

20. MANAL MY, et al. Serum selenium correlations with C-reactive protein, serum calprotectin and disease activity in IBD patients treated with infliximab. World J. of Pharmacy and Pharmac. Sciences, 2015; 4(I.09): 165-179.

21. MOLODECKY N, et al. Increasing incidence and prevalence of the inflammatory bowel diseases with time, based on systematic review. Gastroenterology, 2012; 142(1):46-54.

22. NATIONAL ACADEMY PRESS. Selenium in nutrition, 1983;612'.3924:83-8022.

23. NOTHSTEIN AK, et al. Tracking Se assimilation and speciation through the rice Plant - 506 Nutrient competition, toxicity and distribution. PLoS ONE, 2016;11:1-15. 
24. PINHO M. A Biologia Molecular das Doenças Inflamatórias Intestinais. Rev Bras Coloproct., 2008; 28(1): 119-123.

25. PINTO MAS, et al. Does active Crohn\&\#39; s disease have decreased intestinal antioxidant capacity? J. of Crohn\&\#39; s and Colitis, 2013; 7(9): e358-e366.

26. PROTASIO BKPF et al. Especificidades da apresentação da doença de Crohn na infância. Einstein (São Paulo), 2018; 16(1): eRC4070.

27. SALARI P. New Horizons in Management of Inflammatory Bowel Disease. International J. of Pharmacology, 2011;7: 679-681.

28. SALVIANO FN, et al Perfil socioeconômico e nutricional de pacientes com doença inflamatória intestinal internados em um hospital universitário. Arq Gastroenterol. 2007; 44 (2):99-106.

29. SILVA JF. Selênio, atividade biológica e sua relação com o câncer: uma revisão de literatura. Nutrivisa - Rev de Nutrição e Vigilância em Saúde, 2015;2(1).

30. STEINBRENNER H, et al. Toward Understanding Success and Failures in the Use of Selenium for Cancer Prevention. Antioxid Redox Signal., 2013;19(2):181-91.

31. STOFFANELLER R, MORSE NL. A review of dietary selenium intake and selenium status in Europe and the Middle East. Nutrients, 2015;7:1494-1537.

32. VIARO RS, et al. Importância bioquímica do selênio para o organismo humano: Cien. Biol. e da Sade, Santa Maria, $2001 ; 2(1): 17-21$.

33. WINKEL LHE, et al. Selenium cycling across soil-plant-atmosphere interfaces. Nutrients. 2015; 7(6): 4199-4239.

34. YOON SM. Micronutrient deficiencies in IBD: trivial or crucial? Intest Res., 2016; 14(2):109-110. 